Pacific Journal of Mathematics

THE SPECTRAL APPROACH TO DETERMINING THE 


\title{
THE SPECTRAL APPROACH TO DETERMINING THE NUMBER OF WALKS IN A GRAPH
}

\author{
Frank Harary and Allen J. Schwenk \\ Dedicated to Paul Erdös on his sixtieth birthday, and may \\ he long continue his academic random walks.
}

It is well known that the number of closed walks of length $n$ is simply the $n$th moment of the adjacency matrix. We find similar spectral expressions for unrestricted (either open or closed) walks, and also for walks from any specified starting set of points to another set of terminal points. Knowledge of the number of walks in $G$ may be applied to find the spectrum of the complement of $G$. In conclusion, cyclic and dihedral equivalence relations are defined for closed walks and Burnside's lemma is used to enumerate the number of equivalence classes of both types.

The characteristic polynomial of a graph $G$ is defined as that of its adjacency matrix $A(G)$, and we denote it by

$$
\phi(G ; x)=\prod_{i=1}^{p}\left(x-\lambda_{i}\right) .
$$

Since $A$ is real and symmetric, it has only real roots. This sequence of roots $\lambda_{1} \geqq \lambda_{2} \geqq \cdots \geqq \lambda_{p}$ is called the spectrum of $G$. Another consequence of the symmetry of $A$ is that there exists a unitary matrix that transforms $A$ into Jordan canonical form, and consequently, this form must be a diagonal matrix. Therefore, the minimum polynomial of $A$ can possess no multiple roots, and we write it

$$
\mu(G ; x)=\prod_{i=1}^{d}\left(x-\mu_{i}\right)=\sum_{k=0}^{d} b_{k} x^{k}
$$

where the $\mu_{i}$ are the distinct roots of (1).

An elementary graph theoretic interpretation identifies the trace of $A^{n}$ as the number of closed walks of length $n$ in $G$. But a standard matrix result equates $\operatorname{tr} A^{n}$ to the $n$th moment of $A$ defined as

$$
M_{n}(A)=\sum_{i=1}^{p} \lambda_{i}^{n}
$$

Thus, we have found that the number of closed walks of length $n$ in $G$ is simply the sum of the $n$th powers of the eigenvalues. We might hope that a similar spectral expression could be found for $W_{n}$, the number of arbitrary (either open or closed) walks as length 
$n$ in $G$, and this has been done by Cvetković [2]. We proceed to sketch his results. Since $A$ satisfies $\mu(G ; x)=0$, we find for $n \geqq d$,

$$
A^{n-d} \sum b_{k} A^{k}=\sum b_{k} A^{n-d+k}=0 .
$$

Now $W_{n-d+k}$ is simply the sum of the entries in $A^{n-d+k}$, so summing entries throughout this matrix equation yields

$$
\sum_{k=0}^{d} b_{k} W_{n-d+k}=0 \text {. }
$$

This is a linear homogeneous recurrence relation for the number of walks. But notice that for $1 \leqq i \leqq d$, we may substitute $\mu_{i}^{j}$ for $W_{j}$ to obtain a solution to (5) because $\mu_{i}$ satisfies $\mu(G ; x)=0$. Since this provides $d$ particular solutions which are linearly independent, a general solution must be a linear combination of these solutions. That is, $W_{n}$ must satisfy

$$
W_{n}=\sum_{i=1}^{d} c_{i} \mu_{i}^{n}
$$

where the constants $c_{i}$ are determined by the initial conditions. Cvetkovic [2] defined the main part of the spectrum, denoted $M=$ $\left\{\nu_{1}, \nu_{2}, \cdots, \nu_{m}\right\}$ as the set of those distinct eigenvalues which occur in (6) with a nonzero coefficient. For convenience, we relabel the constants in (6), omitting those constants which are 0 , to obtain

$$
W_{n}=\sum_{i=1}^{m} c_{i} \nu_{i}^{n} .
$$

Thus, Cvetkovic laid the groundwork for solving this problem, but he did not describe how $M$ and the constants $c_{i}$ can be efficiently determined. To do this, we begin by characterizing the main part of the spectrum.

THEOREM 1.2. The following statements are equivalent for a graph $G$.

(1) $M=\left\{\nu_{1}, \nu_{2}, \cdots, \nu_{m}\right\}$ is the main part of the spectrum.

(2) $M$ is the minimum set of eigenvalues the span of whose eigenvectors includes the vector $\beta=(1,1, \cdots, 1)$.

(3) $M$ is the set of those eigenvalues which have an eigenvector not orthogonal to $\beta=(1,1, \cdots, 1)$.

Proof. (1) is equivalent to (2). Since the eigenvectors of $G$ form a basis for $E_{p}$, euclidean $p$-space, $\beta$ can be represented as a linear combination of eigenvectors. For multiple eigenvalues, there is some freedom in choosing the eigenvectors, but we may assume they have been chosen so that at most one vector from each multi- 
ple set is not orthogonal to $\beta$. Consequently, $\beta$ can be expressed as a linear combination of eigenvectors with distinct eigenvalues and nonzero coefficients, say

$$
\beta=\sum_{i=1}^{k} \alpha_{i} \alpha_{i}
$$

Assuming $\alpha_{\imath}$ has eigenvalue $\sigma_{i}$, we observe that

$$
W_{n}=A^{n} \beta \cdot \beta=\sum_{i=1}^{k} \alpha_{i}^{2}\left(\alpha_{i} \cdot \alpha_{i}\right) \sigma_{i}^{n} .
$$

But we have already found the unique expression (7) of this type for $W_{n}$, so we conclude that $k=m$ and $M=\left\{\sigma_{1}, \sigma_{2}, \cdots, \sigma_{k}\right\}$ and that the corresponding constants in (7) and (9) are identical. Thus statements (1) and (2) define the same set, and so (1) is equivalent to (2).

(2) is equivalent to (3). Suppose $\beta=\sum_{i=1}^{m} a_{i} \alpha_{i}$. Then for $1 \leqq$ $i \leqq m, \beta \cdot \alpha_{i}=a_{i}\left(\alpha_{i} \cdot \alpha_{i}\right) \neq 0$, and so every $\nu \in M$ has an eigenvector not orthogonal to $\beta$. Conversely, if each eigenvector $\gamma$ for eigenvalue $\nu$ does not appear among the $\alpha_{i}$ 's, then $\beta \cdot \gamma=\sum \alpha_{i} \alpha_{i} \cdot \gamma=0$. Therefore $\nu \notin M$ implies that all eigenvectors of $\nu$ are orthogonal to $\beta$, so (2) is equivalent to (3).

Let the neighborhood of $v$, denoted $N(v)$, be the set of points adjacent to $v$. In [5, §3] we call a partition $V_{1} \cup V_{2} \cup \cdots \cup V_{m}$ of the points of graph $G$ equitable if for all $i, j$ and for all $u, v \in V_{i}$

$$
\left|N(u) \cap V_{j}\right|=\left|N(v) \cap V_{j}\right| \text {. }
$$

For example, the similarity classes of points formed by the automorphism group of $G$ constitute an equitable partition. We associate with each equitable partition a matrix $T$ defined by

$$
t_{i j}=\left|N(u) \cap V_{j}\right|
$$

where $u \in V_{i}$. We conjecture that there is a fourth statement equivalent to the three in Theorem 1.

Conjecture (4). Let the matrix $T$ be obtained from an equitable partition of smallest size. Then $T$ is an $m \times m$ matrix and $M$ is just the set of eigenvalues of $T$.

If $\alpha_{1}, \alpha_{2}, \cdots, \alpha_{p}$ form an orthonormal basis, the theorem permits us to rewrite (7) in the form

$$
W_{n}=\sum_{i=1}^{p}\left(\beta \cdot \alpha_{i}\right)^{2} \lambda_{i}^{n}
$$

That is, $\lambda_{i} \in M$ if and only if $\beta \cdot \alpha_{i} \neq 0$, and so the constants $c_{i}$ of (7) are just the squares of the dot products $\left(\beta \cdot \alpha_{i}\right)$. 
It is easy to see how to adjust (9) to obtain the number of walks of length $n$ from any subset $B$ of possible starting points to a second subset $C$ of permitted terminal points. Let $\gamma$ be the vector whose $v_{i}$ component is 1 or 0 according as $v_{i}$ is or is not in $B$. Let $\delta$ be the corresponding vector determined by $C$.

Corollary 1a. The number $W_{n}(B, C)$ of walks of length $n$ from any set $B$ to another set $C$ is

$$
W_{n}(B, C)=A^{n} \gamma \cdot \delta=\sum_{i=1}^{p}\left(\gamma \cdot \alpha_{i}\right)\left(\delta \cdot \alpha_{i}\right) \lambda_{i}^{n} .
$$

Since it is well known (see for example Collatz and Sinogowitz [1]) that the largest eigenvalue $\lambda_{1}$ (also called the spectral radius) has a nonnegative eigenvector, we see that $c_{1}=\left(\beta \cdot \alpha_{1}\right)^{2}>0$, and we quickly obtain another result of Cvetković [3].

\section{THEOREM 2. The spectral radius is given by}

$$
\lambda_{1}=\lim _{n \rightarrow \infty} \sqrt[n]{W_{n} / p}
$$

Notice that since $p$ remains fixed as $n$ increases, it has no affect on the limit. It has been included simply to make the individual terms identical to the $n$th root of what Cvetkovic calls "the dynamic average degree". In particular when $n=1$, notice that $W_{n} / p$ is just the usual average degree.

In [5] we discussed the relation between spectra and various graphical operations. We can now determine $\phi(\bar{G})$ and hence the spectrum of the complement. The ordinary generating function for the number of walks of length $n$ is readily found from (7) to be

$$
W(x)=\sum_{n=0}^{\infty} W_{n} x^{n}=\sum_{i=1}^{m} c_{i} /\left(1-\nu_{i} x\right) .
$$

Using this function, Cvetković [2] was able to express the characteristic polynomial of the complement in terms of $\phi(G)$.

THEOREM 3. The characteristic polynomial of $\bar{G}$ is

$$
\phi(\bar{G} ; x)=(-1)^{p} \phi(G ;-1-x)(1-W(-1 /(1+x)) /(1+x)) .
$$

For example, if $G$ is regular, $W(x)$ has the particularly simple form

$$
W(x)=p /(1-r x) \text {. }
$$

Substituting this into (16), we find that for a regular graph $G$, 


$$
\phi(\bar{G} ; x)=(-1)^{p} \phi(G ;-1-x)(1+r-p+x) /(1+r+x) .
$$

By inspection, we see that if $\left(r, \lambda_{2}, \lambda_{3}, \cdots, \lambda_{p}\right)$ is the spectrum of $G$, then the spectrum of $\bar{G}$ is $\left(p-1-r,-1-\lambda_{2},-1-\lambda_{3}, \cdots,-1-\lambda_{p}\right)$. Notice that most of the eigenvalues are -1 minus the corresponding eigenvalue of $G$. This is in fact a special case of the following result.

THEOREM 4. Let $\alpha$ be an eigenvector of $G$ with eigenvalue $\lambda$ and suppose $\alpha$ is orthogonal to $\beta=(1,1, \cdots, 1)$. Then $\alpha$ is an eigenvector of $\bar{G}$ with eigenvalue $-1-\lambda$.

Proof. Since $\bar{A}=J-I-A$, we see that

$$
\bar{A} \alpha=J \alpha-I \alpha-A \alpha \doteq 0 \alpha-\alpha-\lambda \alpha=(-1-\lambda) \alpha .
$$

In other words, the nonmain eigenvalues of $\bar{G}$ are easily found from those of $G$. The difficulty is to find the main eigenvalues. Theorem 3 accomplishes this in general. If the Conjecture (4) holds, there is another way to find the main part of the spectrum of $\bar{G}$. Namely, the equitable partition found for $G$ is also equitable for $\bar{G}$. The corresponding matrix $\bar{T}$ is given by

$$
\bar{t}_{i j}=\left|V_{j}\right|-t_{i j}-\delta_{i j},
$$

where $\delta_{i j}$ is the Kroneker delta function. Thus, the main eigenvalues are the roots of $\bar{T}$ and the nonmain eigenvalues are readily found from Theorem 4.

We conclude by returning to the enumeration of closed walks. Each walk counted by (3) has a specified starting point. Thus the walks $v_{1} v_{2} \cdots v_{n-1} v_{n} v_{1}$ and $v_{2} v_{3} \cdots v_{n} v_{1} v_{2}$ are different walks (even though they traverse the same points in the same relative order) because one starts at $v_{1}$ and the other starts at $v_{2}$. We define two closed walks to be cyclic equivalent if one can be obtained from the other by rotating an initial segment to the end of the walk. One might first suspect that each equivalence class has $n$ walks in it, but that is not always true. For example, with $n=6$, the equivalence class of $v_{1} v_{2} v_{3} v_{1} v_{2} v_{3} v_{1}$ has just three walks. Thus, we ask what is $C_{n}(G)$, the number of cyclic equivalence classes of closed walks of length $n$ in $G$ ? Let $\phi(n)$ denote the Euler phi-function ${ }^{1}$ and let $(i, n)$ be the greatest common divisor of $i$ and $n$.

Theorem 5. $C_{n}(G)=1 / n \sum_{d \mid n} \phi(n / d) \sum_{j=1}^{p} \lambda_{j}^{d}$.

Proof. We apply Burnside's lemma (see [4, p. 181]) to the ${ }^{1}$ This is a minor $\sin$ of notation since $\phi(G ; x)$ is the characteristic polynomial. 
cyclic group of order $n$ acting on closed walks of length $n$, observing that a walk $W$ is "fixed" under a rotation of $i$ positions if and only if $W$ consists of a closed walk of length $(i, n)$ repeated $n /(i, n)$ times. Thus

$$
C_{n}(G)=\frac{1}{n} \sum_{i=1}^{n} \operatorname{tr} A^{(i, n)}
$$

If we let $d=(i, n)$, we may regroup (21) as a sum over the divisors of $n$. Each $d$ occurs as an exponent as often as it is the greatest common divisor of $n$ and some $i$, which is just $\dot{\phi}(n / d)$ times. Therefore,

$$
C_{n}(G)=\frac{1}{n} \sum_{d i n} \dot{\phi}(n / d) \operatorname{tr} A^{d},
$$

and the theorem is verified by substitution from (3).

Another equivalence relation, called dihedral equivalence, is obtained if we permit reversals in addition to rotations. That is, we consider the dihedral group $D_{n}$ acting on closed walks of length $n$. We let $D_{n}(G)$ denote the number of these dihedral equivalence classes.

CoROllary 5a.

$$
D_{n}(G)=\left\{\begin{array}{l}
\frac{1}{2 n} \sum_{d \mid n} \phi(n / d) \sum_{j=1}^{p} \lambda_{j}^{d} \text { if } n \text { is odd } \\
\frac{1}{2 n} \sum_{d ; n} \phi(n / d) \sum_{j=1}^{p} \lambda_{j}^{d}+\frac{1}{4} \sum_{j=1}^{m} c_{j} \nu_{j}^{n / 2} \text { if } n \text { is even } .
\end{array}\right.
$$

Proof. The rotations in $D_{n}$ are handled just as they were in $C_{n}$ above, so we need only account for the $n$ reflections in $D_{n}$. A reflection that interchanges two consecutive points in the walk cannot fix any closed walk because two consecutive points must be distinct since $G$ has no loops. But when $n$ is odd, each reflection does interchange two adjacent points, and so we obtain the first half of (23). When $n$ is even, on the other hand, $n / 2$ reflections interchange consecutive points while the other $n / 2$ reflections do not. These latter reflections "fix" a walk if and only if it consists of an arbitrary walk of length $n / 2$ followed by its reversal. Thus, the reflections contribute $n / 2(1 / 2 n) W_{n / 2}$ when $n$ is even, and so (23) is verified.

\section{REFERENCES}

1. L. Collatz and U. Sinogowitz, Spectra of finite graphs, Abh. Math. Sem. Univ. Hamburg, 21 (1957), 64-77. 
2. D. M. Cvetković, The generating function for variations with restrictions and paths of the graph and self-complementary graphs, Publ. Elektrotehn. Fak. Univ. Beograd, Ser. Mat. Fiz., Nos. 320-328 (1970), 27-34.

3. - Graphs and their spectra, Publ. Elektrotehn. Fak. Univ. Beograd, Ser. Mat. Fiz., Nos. $354-356$ (1971), 1-50.

4. F. Harary, Graph Theory, Addison-Wesley, Reading, 1969.

5. A. J. Schwenk, Computing the characteristic polynomial of a graph, Graphs and Combinatorics (R. Bari and F. Harary, eds.), Springer, Berlin, (1974), 153-172.

Received March 25, 1976. Research supported in part by grant 73-2502 from the Air Force Office of Scientific Research.

The UnIVERSity OF Michigan

ANN ARBor, MI 48104 



\section{PACIFIC JOURNAL OF MATHEMATICS}

\section{EDITORS}

RICHARD ARENS (Managing Editor)

University of California

Los Angeles, CA 90024

Charles W. Curtis

University of Oregon

Eugene, OR 97403

C. C. MOORE

University of California

Berkeley, CA 94720
J. DUGUNDJI

Department of Mathematics

University of Southern California

Los Angeles, CA 90007

R. FINN and J. MILGRAM

Stanford University

Stanford, CA 94305

\section{ASSOCIATE EDITORS}

\section{E. F. BeCKENBACH}

B. H. NeumanN

F. WOLF

K. YoSHIDA

\section{SUPPORTING INSTITUTIONS}

\author{
UNIVERSITY OF SOUTHERN CALIFORNIA \\ STANFORD UNIVERSITY \\ UNIVERSITY OF HAWAII \\ UNIVERSITY OF TOKYO \\ UNIVERSITY OF UTAH \\ WASHINGTON STATE UNIVERSITY \\ UNIVERSITY OF WASHINGTON
}

The Supporting Institutions listed above contribute to the cost of publication of this Journal, but they are not owners or publishers and have no responsibility for its content or policies.

Mathematical papers intended for publication in the Pacific Journal of Mathematics should be in typed form or offset-reproduced, (not dittoed), double spaced with large margins. Please do not use built up fractions in the text of the manuscript. However, you may use them in the displayed equations. Underline Greek letters in red, German in green, and script in blue. The first paragraph or two must be capable of being used separately as a synopsis of the entire paper. Items of the bibliography should not be cited there unless absolutely necessary, in which case they must be identified by author and journal, rather than by item number. Manuscripts, in triplicate, may be sent to any one of the editors. Please classify according to the scheme of Math. Reviews, Index to Vol. 39. All other communications should be addressed to the managing editor, or Elaine Barth, University of California, Los Angeles, California, 90024.

50 reprints to each author are provided free for each article, only if page charges have been substantially paid. Additional copies may be obtained at cost in multiples of 50 .

The Pacific Journal of Mathematics is issued monthly as of January 1966. Regular subscription rate: $\$ 72.00$ a year (6 Vols., 12 issues). Special rate: $\$ 36.00$ a year to individual members of supporting institutions.

Subscriptions, orders for numbers issued in the last three calendar years, and changes of address should be sent to Pacific Journal of Mathematics, P.O. Box 969, Carmel Valley, CA 93924, U.S.A. Older back numbers obtainable from Kraus Periodicals Co., Route 100, Millwood, NY 10546.

PUBLISHED BY PACIFIC JOURNAL OF MATHEMATICS, A NON-PROFIT CORPORATION

Printed at Kokusai Bunken Insatsusha (International Academic Printing Co., Ltd.). 8-8, 3-chome, Takadanobaba, Shinjuku-ku, Tokyo 160, Japan. 


\section{Pacific Journal of Mathematics}

\section{Vol. 80, No. $2 \quad$ October, 1979}

K. Adachi, On the multiplicative Cousin problems for $N^{p}(D) \ldots \ldots \ldots \ldots 297$

Howard Banilower, Isomorphisms and simultaneous extensions in $C(S) \ldots 305$

B. R. Bhonsle and R. A. Prabhu, An inversion formula for a distributional

finite-Hankel-Laplace transformation ................... 313

Douglas S. Bridges, Connectivity properties of metric spaces.......... 325

John Patton Burgess, A selection theorem for group actions ........... 333

Carl Claudius Cowen, Commutants and the operator equations

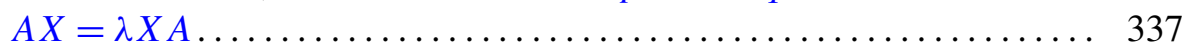

Thomas Curtis Craven, Characterizing reduced Witt rings. II .......... 341

J. Csima, Embedding partial idempotent d-ary quasigroups ............ 351

Sheldon Davis, A cushioning-type weak covering property ............ 359

Micheal Neal Dyer, Nonminimal roots in homotopy trees ............. 371

John Erik Fornaess, Plurisubharmonic defining functions ........... 381

John Fuelberth and James J. Kuzmanovich, On the structure of finitely

generated splitting rings .......................... 389

Irving Leonard Glicksberg, Boundary continuity of some holomorphic

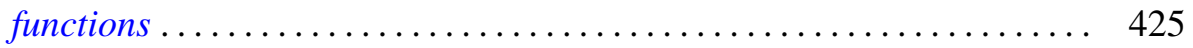

Frank Harary and Robert William Robinson, Generalized Ramsey theory.

IX. Isomorphic factorizations. IV. Isomorphic Ramsey numbers .......

Frank Harary and Allen John Carl Schwenk, The spectral approach to determining the number of walks in a graph...........

David Kent Harrison, Double coset and orbit spaces ..... . .

Shiro Ishikawa, Common fixed points and iteration of commuting

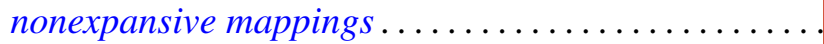

Philip G. Laird, On characterizations of exponential polynomials ........ 503

Y. C. Lee, A Witt's theorem for unimodular lattices ...........

Teck Cheong Lim, On common fixed point sets of commutative

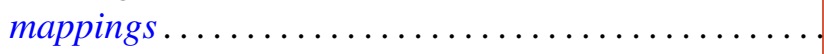

R. S. Pathak, On the Meijer transform of generalized functions ...

T. S. Ravisankar and U. S. Shukla, Structure of $\Gamma$-rings . . .

Olaf von Grudzinski, Examples of solvable and nonsolvable convolution

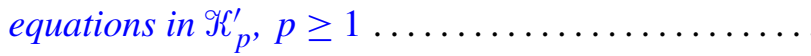

